\title{
Intra-abdominal chylovenous bypass treats retroperitoneal lymphangiomatosis
}

\author{
Courtney Chen BS ${ }^{1} \odot \mid$ Sung-Yu Chu MD² $\mid$ Chieh Lin MD, PhD ${ }^{3} \mid$ Ken-Hao Liu MD \\ Ming-Huei Cheng MD, MBA, FACS ${ }^{5,6,7}$ (1)
}

${ }^{1}$ UC San Diego School of Medicine, San Diego, California

${ }^{2}$ Department of Medical Imaging and Intervention, Chang Gung Memorial Hospital, Taoyuan, Taiwan

${ }^{3}$ Department of Nuclear Medicine, Chang Gung Memorial Hospital, Taoyuan, Taiwan

${ }^{4}$ Department of General Surgery, Chang Gung Memorial Hospital, Taoyuan, Taiwan

${ }^{5}$ Division of Reconstructive Microsurgery, Department of Plastic and Reconstructive Surgery, Chang Gung Memorial Hospital, College of Medicine, Chang Gung University, Taoyuan, Taiwan

${ }^{6}$ Center for Tissue Engineering, Chang Gung Memorial Hospital, Taoyuan, Taiwan

${ }^{7}$ Department of Plastic Surgery, University of Michigan, Ann Arbor, Michigan

\section{Correspondence}

Ming-Huei Cheng, MD, MBA, FACS Division of Reconstructive Microsurgery, Department of Plastic and Reconstructive Surgery, Chang Gung Memorial Hospital, College of Medicine, Chang Gung University,

5 Fu-Hsing Street, Kueishan, Taoyuan 333, Taiwan.

Email: minghueicheng@gmail.com and minghuei@cgmh.org.tw

\begin{abstract}
Background: Retroperitoneal lymphangiomatosis ( $R L$ ) is a rare form of primary lymphedema featuring aberrant retroperitoneal lymphatic proliferation. It causes recurrent cellulitis, repeated interventions, and poor life quality. This study aimed to investigate proper diagnositc criteria and surgical outcomes for RL with extremity lymphedema.

Methods: Between 2012 and 2018, 44 primary lower-extremity lymphedema cases received lymphoscintigraphy, magnetic resonance imaging, and single-photon electron computed tomography to detect RL. RL patients underwent vascularized lymph node transfers (VLNT) for extremity lymphedema and intra-abdominal side-toend chylovenous bypasses (CVB) for chylous ascites. Complications, CVB patency, and quality of life were evaluated postoperatively.

Results: Six RL patients (mean age of 30.3 years) had chylous ascites with five had lower-extremity lymphedema. All CVBs remained patent, though one required reanastomosis, giving a $100 \%$ patency rate. Four unilateral and one bilateral extremity lymphedema underwent six VLNTs with $100 \%$ flap survival. Patients reported improved quality of life $(P=0.023)$, decreased cellulitis incidence $(P=0.041)$, and improved mean lymphedema circumference $(P=0.043)$. All patients resumed a normal diet and activity.

Conclusions: Evaluating primary lower-extremity lymphedema patients with MRI and SPECT could reveal a $13.6 \%$ prevalence of $R L$ and guide treatment of refractory extremity lymphedema. Intra-abdominal CVB with VLNT effectively treated RL with chylous ascites and extremity lymphedema.
\end{abstract}

\section{KEYWORDS}

chylovenous bypass, extremity lymphedema, retroperitoneal lymphangiomatosis, vascularized lymph node transfer

\section{1 | INTRODUCTION}

Lymphangiomatosis is a rare condition of unknown incidence with diffuse involvement of all tissue types through hyperproliferation of lymphatic vasculature, causing multiple lymphangiomas. ${ }^{1,2}$ This condition affects more children than adults. ${ }^{2,3}$ Lymphangiomatosis is classified as either generalized lymphangiomatosis or Gorham Stout syndrome, with the former primarily involving soft tissue and viscera and secondary osseous involvement, while the latter features lymphovascular proliferation within bones and occasional soft tissue/ viscera involvement. ${ }^{3,4}$ Major complications of lymphangiomatosis include chylothorax, chylous pericardial effusions, and chylous 
ascites. ${ }^{5,6}$ Chylous ascites has a poor prognosis without hope of spontaneous resolution. Persistent loss of chyle can also cause nutritional imbalances. ${ }^{7,8}$ Retroperitoneal lymphangiomatosis (RL) with concomitant lower-limb lymphedema has also been reported. ${ }^{9}$ Current standards of care for these complications largely involve symptomatic relief by thoracentesis, pleurodesis, or paracentesis and combined therapy for any lymphedema. ${ }^{10}$

While medical and surgical management of RL exist, not all are effective or even resolve secondary chylous ascites or lowerextremity lymphedema. There was limited evidence for proposed medical treatments such as bisphosphonate, sirolimus, interferon, and radiotherapy. ${ }^{2,7,8}$ There was also a paucity in interventional methods as well, especially for chylous ascites.

Kinmonth first described a series of patients with lower-extremity lymphedema caused by ectatic lymphatic channels in the abdomen, involving the retroperitoneum to the mesentery/intestines. Treatment included repairing leaks, ligating incompetent channels, and resection (eg, intestinal resection, hysterectomy). ${ }^{9}$ Sclerotherapy has also been proposed with varied reception. ${ }^{8,10-12}$ Noel et al reported 4 chylovenous anastomoses out of 26 surgical procedures in 30 patients with primary chylous disorders. ${ }^{10}$ Campisi et al opted for exeresis, $\mathrm{CO}_{2}$ laser treatments, and chylovenous/lymphovenous shunts in 12 patients with primary chylous ascites (not all had RL). ${ }^{8}$ Their shunt techniques varied, with direct lymphatic duct repair to the subcutaneous venous anastomosis, chylous channel/cyst-to-venous shunt anastomosis, or lymphatic-to-venous anastomosis using vein grafts. Yet, anastomoses details were not reported, such as the level a technique was performed at or specific technique outcomes.,

To address such issues, our study aimed to investigate standardized diagnostic criteria and surgical outcomes of intra-abdominal chylovenous bypass (CVB) with vascularized lymph node transfer (VLNT) for RL with chylous ascites and concomitant lower-extremity lymphedema.

\section{2 | MATERIALS AND METHODS}

Between 2012 and 2018, patients who presented with primary lymphedema or chylous discharge were retrospectively reviewed with institutional review board approval (IRB: 201801591B0) at Linkou Chang Gung Memorial Hospital. Each patient diagnosed with chylous ascites underwent an assessment to discover the etiology, ranging from clinical measurements to imaging to detection of albumin levels. Ultrasound Doppler and computed tomography angiography (CTA) were utilized to rule out any proximal vascular lesions and to evaluate the patency of any VLNT for lower-extremity lymphedema. ${ }^{13}$ Lymphoscintigraphy was performed, and Taiwan Lymphoscintigraphy Staging was used to interpret findings of partial or total lymphatic obstruction of the lower extremities. ${ }^{14}$ Magnetic resonance imaging (MRI) was performed to confirm extravasation of contrast into the peritoneal cavity and to visualize aberrant lymphatic tracts in the soft tissue. Since 2018, single-photon emission computed tomography (SPECT) was added to support the presence of abnormal retroperitoneal lymphatic tracts and evaluate disease extent. Indocyanine green (ICG) lymphography was performed in cases of mild extremity lymphedema.

When a patient was confirmed to have extremity lymphedema of Cheng's Grade II-IV, VLNT was suggested to first relieve the extremity lymphedema, followed by CVB to address the chylous ascites. The clinical rationale for performing VLNT instead of lymphovenous anastomosis (LVA) for extremity lymphedema was that prior studies had demonstrated that LVA is indicated for cases of Cheng Lymphedema Grade I-II lymphedema while VLNT is indicated for Grade II-IV lymphedema. ${ }^{14}$ Donor VLNT flaps were selected from the omentum or submental basins. ${ }^{15}$ Patients were monitored immediately after CVB in a microsurgical ICU ward for 3 to 5 days before being transferred to a regular ward. Patients were followed postoperatively and evaluated for changes in limb circumference, chylous ascites, patency of the CVB by CTA, complications, body weight, and nutrition. Patients were also asked to rate their quality of life (QoL) using a visual analog score scaled 1 to 10 at their initial and latest clinical visit, with 1 being the poorest and 10 being the best.

\section{1 | Technique for VLNT}

The preoperative evaluation and technique of VLNT have previously been described by the authors. ${ }^{16-18}$ Before the intra-abdominal CVB, the patency and effectiveness of any prior VLNT were tested by Doppler ultrasound and CTA. If the VLNT demonstrated impaired venous flow, it was revised, and the pedicle was confirmed to be patent with the adequate flow at the time of intra-abdominal CVB.

\subsection{Techniques for intraabdominal chylovenous bypass}

The abdomens of patients were opened with a $15 \mathrm{~cm}$ incision for an exploratory laparotomy by a general surgeon (K-H Liu). Surgical exploration then identified the potential site of damaged lymphatics responsible for chyle leakage in the RL soft tissue, as evidenced by focal milky fluid exudate. The precise site of leakage was confirmed under a microscope (Zeiss OPMI Pentero 800, Jena, Germany) at 20-25× magnification, with the location typically being between the level of the umbilicus and the anterior superior iliac spine around the ureter and internal iliac artery. A $4 \mathrm{~mm}$ diameter hole was created by bipolar cautery in lymphangiomatosis soft tissue as a controlled outlet for chyle. The ovarian vein was then identified in relation to the outlet. If the distance between the ovarian vein and outlet could not be bridged without tension, branches from the internal iliac vein were then identified as alternative recipient veins and dissected free. The designated recipient vein was separated distally. The distal end of the vein was then anastomosed to the artificial hole in a side-to-end fashion with 8 to 12 stitches using 9-0 nylon. ICG $(0.1 \mathrm{ml})$ was injected into the distal site of RL. Fluorescent flow was seen under microscopy to travel from the lymphangiomatosis through the anastomosis and to the recipient vein, verifying intraoperative CVB patency. 


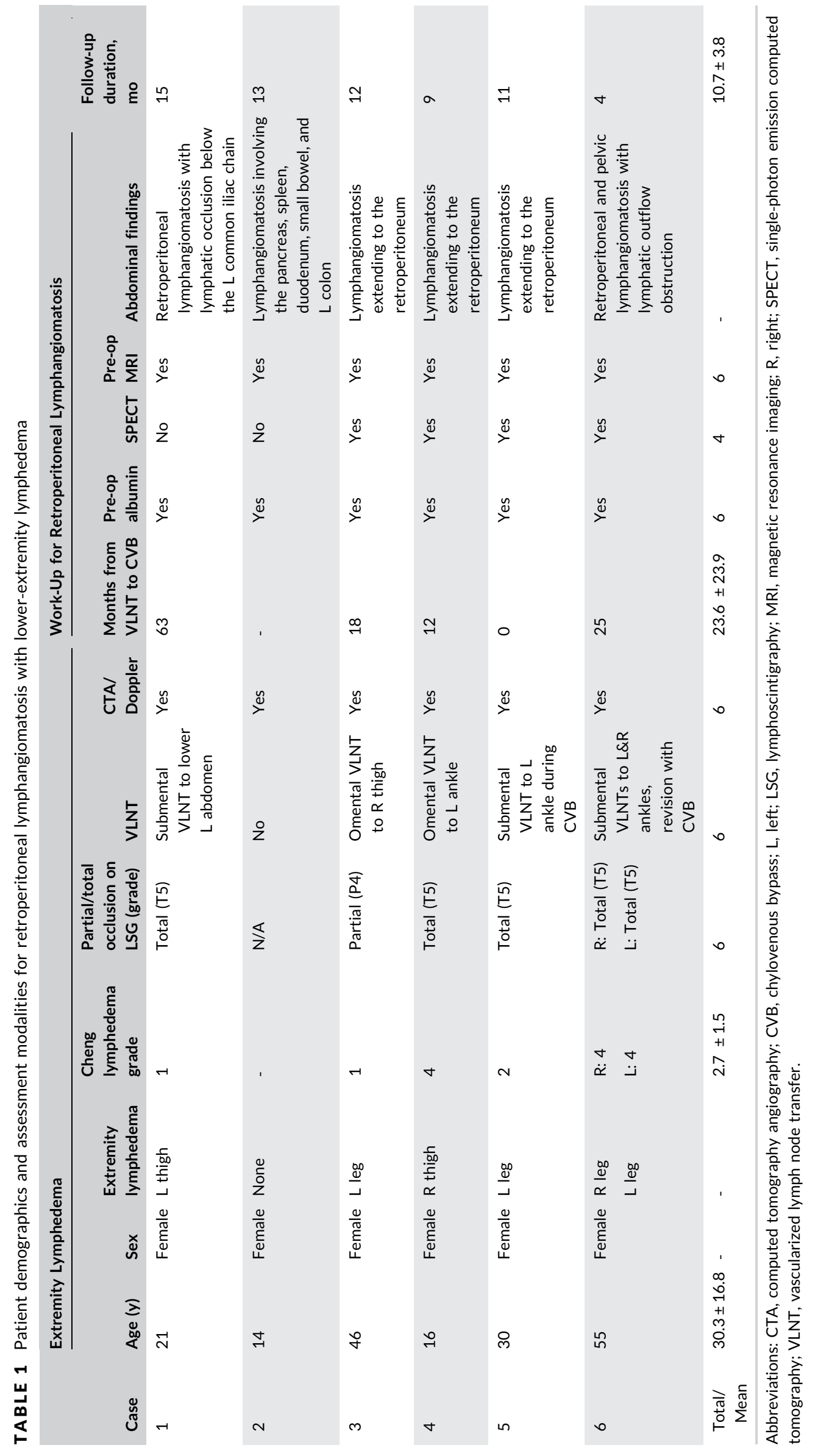




\section{3 | Statistical analysis}

Data before and after VLNT and CVB, including data on clinical monitoring of chylous ascites, body weight, subjective QoL measures, and circumferential sizes of limbs, were collected and analyzed with IBM SPSS Statistics 20 statistical software (IBM Corporation, Armonk, NY). Data were reported as the mean \pm standard deviation, and the circumference of the lower limbs measured $15 \mathrm{~cm}$ proximal to the patella, $15 \mathrm{~cm}$ distal to the patella, and $10 \mathrm{~cm}$ proximal to the ankle. Comparisons between groups were performed using the Wilcoxon signed rank test. A two-tailed $P$ value $<0.05$ was considered statistically significant.

\section{RESULTS}

A total of 44 primary lymphedema patients were evaluated at Linkou Chang Gung Memorial Hospital between 2012 and 2018. Six of the forty-four patients were diagnosed with RL (incidence: 13.6\%). There were seven primary lymphedema patients who received abdominal
SPECT and MRI with results possibly indicative of RL, prompting exploratory laparotomy. Exploration found $\mathrm{RL}$ in six of the seven patients. The specificity of SPECT plus MRI was $85.7 \%(6 / 7)$ in determining $\mathrm{RL}$ in primary lymphedema patients.

The average age of patients was 30.3 years (range $14-55$ years) (Table 1). All were female. Four patients presented with unilateral lower-extremity lymphedema, with two patients presenting with chyle leakage in the thigh, and one presenting with bilateral lowerextremity lymphedema (Figure 1, Table 1). The mean preoperative Cheng Lymphedema Grade of the six lymphedematous limbs was 2.7 (range 1-4). Of the six patients, four underwent five VLNTs before CVB, one underwent CVB only, and one underwent VLNT simultaneously with the CVB (Figure 1). The average time between CVB and VLNT was $23.6 \pm 23.9$ months (Table 1). Ultrasound Doppler studies showed patency and good flow in all pedicle anastomoses in the six VLNTs without stenosis, and CTA demonstrated uncompromised VLNTs. All six patient MRIs revealed chylous ascites, and lymphoscintigraphy confirmed partial or total obstruction in the lower extremity (Figure 2).
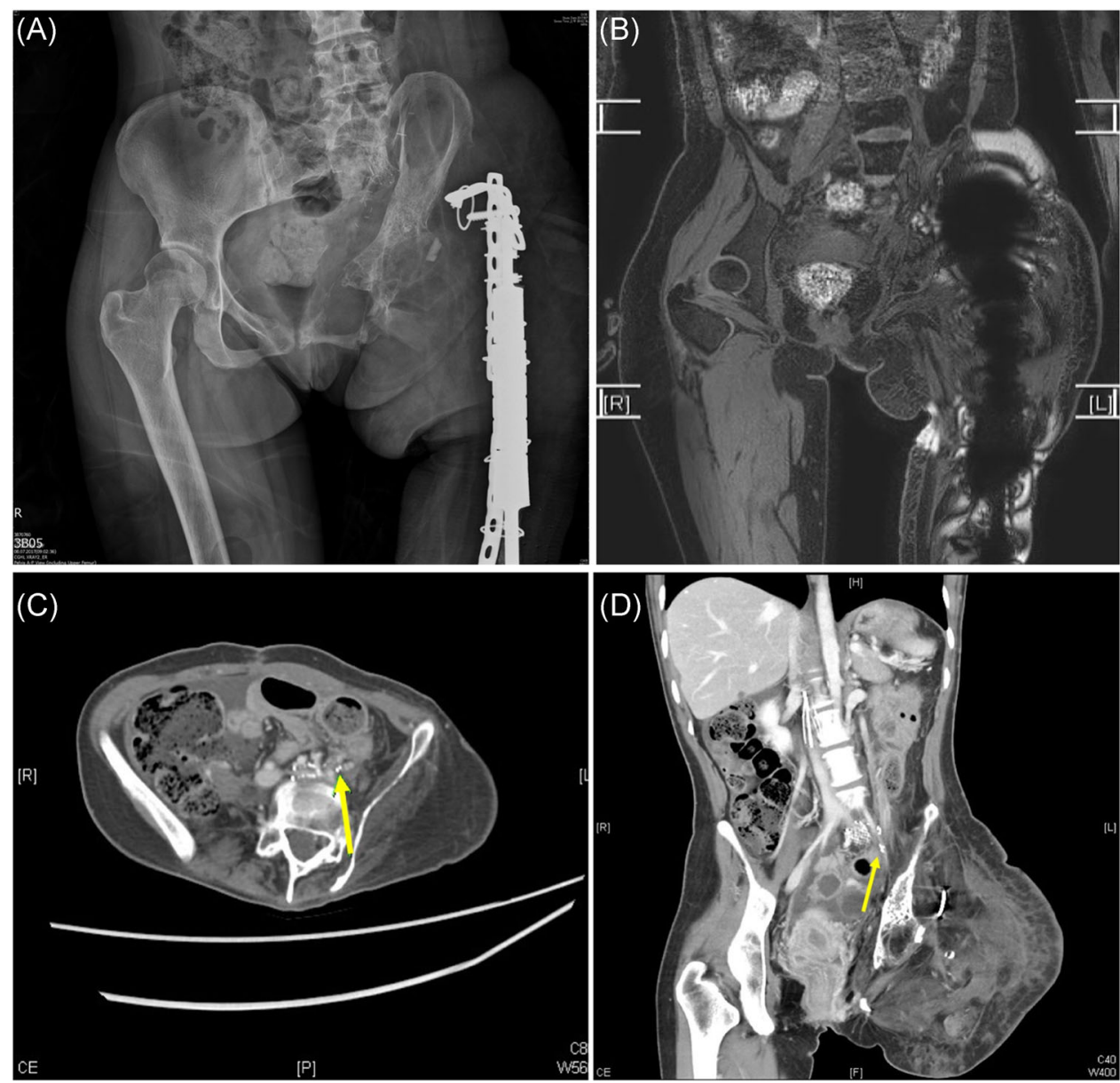

FIGURE 1 Case 1: Initial imaging and follow-up imaging. A, Pelvic X-ray revealed left femoral shaft and head fractures with an internal prosthesis and wiring fixation of a left iliac osteolytic fracture. B, MRI showed extravasation of contrast media to the left pelvis, left femoral area, and bilateral paraspinal regions, consistent with retroperitoneal lymphangiomatosis. The soft tissue of the left pelvis and thigh showed characteristic honeycomb lymphedematous fibrosis. C,D, Computed tomography angiography revealed a patent chylovenous bypass (indicated by the yellow arrow) at the left iliac region without chylous ascites at 3 weeks (C, coronal section) and 3 months (D, sagittal section) postoperatively [Color figure can be viewed at wileyonlinelibrary.com] 

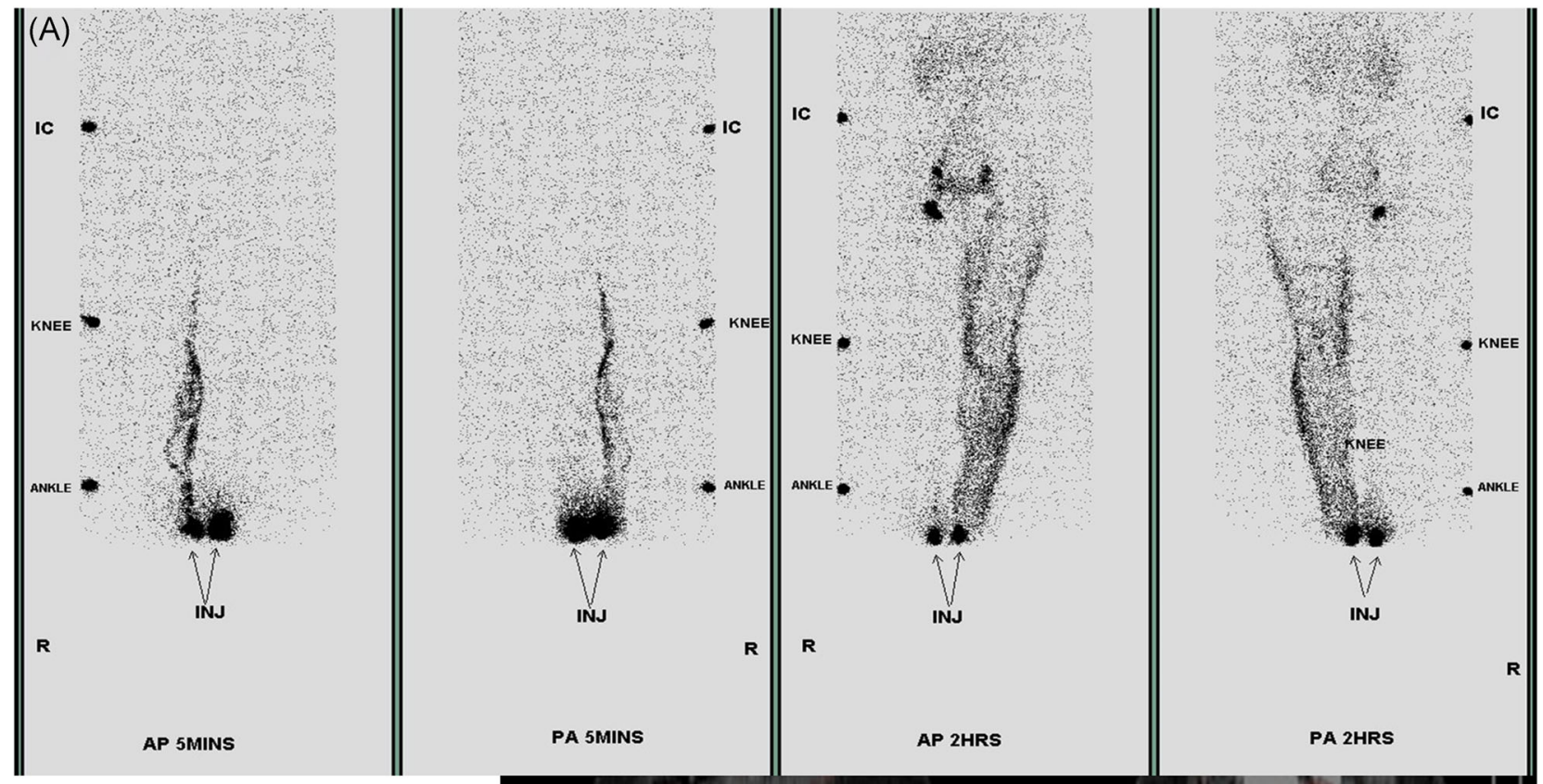

(B)

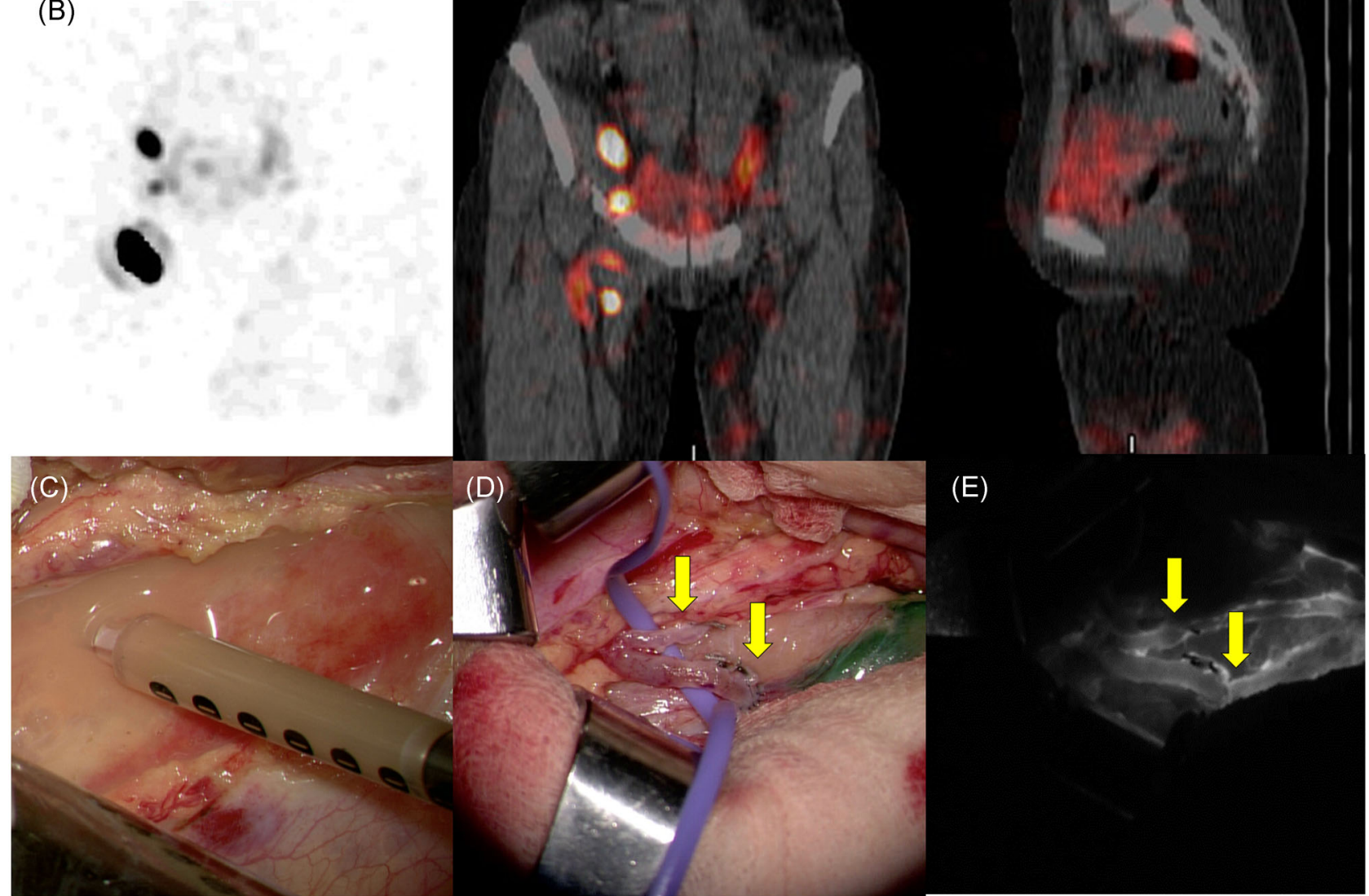

FIGURE 2 Case 5: Lymphoscintigraphy, single-photon emission computed tomography, and intraoperative images with indocyanine green confirmation. A, Anterior and posterior planar imaging of the lower extremities at 5 minutes and 2 hours post subcutaneous injection of $0.5 /$ $0.5 \mathrm{mCi}$ Tc-99m phytate. Lymphoscintigraphy revealed reduced inguinal/pelvic lymph nodes uptake and dermal backflow over the entire left lower limb with no major abnormalities in the right lower limb in comparison. B, Accumulated tracer along the left iliac vessels/paraaortic area was suggestive of possible lymphangiomatosis with dilated lymphatic ducts in single-photon emission computed tomography. C, Chyle (aspirated by a syringe) was actively leaking from the soft tissue of the retroperitoneal lymphangiomatosis intraoperatively at the prespinal area. D, Two chylovenous bypasses were performed with 9-0 Nylon in a side-to-end fashion (yellow arrows indicating side-to-end anastomosis sites). E, Intraoperative ICG imaging confirmed patency of the chylovenous bypasses. ICG, indocyanine green [Color figure can be viewed at wileyonlinelibrary.com] 
The average albumin level changed from $3.1 \pm 0.6 \mathrm{~g} / \mathrm{dL}$ pre-CVB to $3.3 \pm 0.7 \mathrm{~g} / \mathrm{dL}$ post-CVB $(P=0.500)$. The mean body weights pre- and post-CVB were $56.7 \pm 14.1 \mathrm{~kg}$ and $53.5 \pm 10.7 \mathrm{~kg}$, respectively $(P=0.07)$. Mean limb circumference after VLNT and CVB significantly improved by $4.2 \pm 2.2 \mathrm{~cm} \quad(P=0.043)$, and improved $2.6 \pm 2.9 \mathrm{~cm}$ above the knee $(P=0.068), 5.9 \pm 4.7 \mathrm{~cm}$ below the knee $(P=0.042)$, and $3.3 \pm 2.2 \mathrm{~cm}$ above the ankle $(P=0.078)$. Mean cellulitis incidence significantly declined from $1.9 \pm 1.8$ to $0.1 \pm 0.4$ times/year postoperatively $(P=0.04)$ (Table 2$)$.

Immediate patency of the CVB was verified in all patients through intraoperative ICG microscopy (Figure 2). The average cohort length of stay after CVB was 14.2 \pm 4.1 days (Table 3 ). Before discharge, all patients had $<30 \mathrm{~mL} /$ day of chylous output from abdominal drains. No abdominal drains needed to be reinserted. One patient experienced post-CVB complications of perforation of the jejunum and thrombosis of the chylovenous anastomosis site, which required resection with primary repair of the jejunum and thrombectomy with re-anastomosis of the CVB, respectively. By discharge, all patients were able to return to normal activity. Patients were able to tolerate a regular diet instead of a low-fat diet by 6 months postoperatively without recurrence of chylous ascites (Table 3 ). The resolution of chylous ascites in addition to patency of the CVB was verified through CTA at postoperative visits for all patients. In addition, during follow-up surveillance, five of six patients were able to discontinue the use of compression garments for lymphedematous extremities by 6 months, with one patient continuing to require compression garments for 8 to 10 hours during the day for thigh lymphedema (Figure 4) (Table 3). The mean QoL score significantly improved from $3.4 \pm 0.8$ at before the operation to $5.7 \pm 1.3$ after the operation $(P=0.023)$.

\section{DISCUSSION}

Utilization of abdominal MRI was initiated due to the VLNTrefractory nature of extremity lymphedema with additional abdominal symptoms. MRI demarcated soft tissue changes and lymphatic proliferation of lymphangiomatosis. SPECT in the last four patients helped further elucidate the disease extent. Overall, lymphoscintigraphy, MRI, and SPECT were able to definitively demonstrate chylous ascites secondary to RL (Figure 3 ).

Once VLNT was demonstrated to be inadequate for the concomitant lower-extremity lymphedema, CVB was performed as a potential physiologic treatment for the extravasating lymph causing the lymphedema and chylous ascites. The CVB procedure appeared to be effective in treating the chylous ascites, as supported by the absence of clinically detectable ascites with imaging validation in all six patients. In addition, after CVB, there was an additional significant reduction in leg circumference below the knee beyond the VLNTrelated reduction $(P=0.042)$. The only exception to this trend was patient 5 , who removed her compression garments only during her pre-CVB measurements and therefore had an unknown true baseline. 

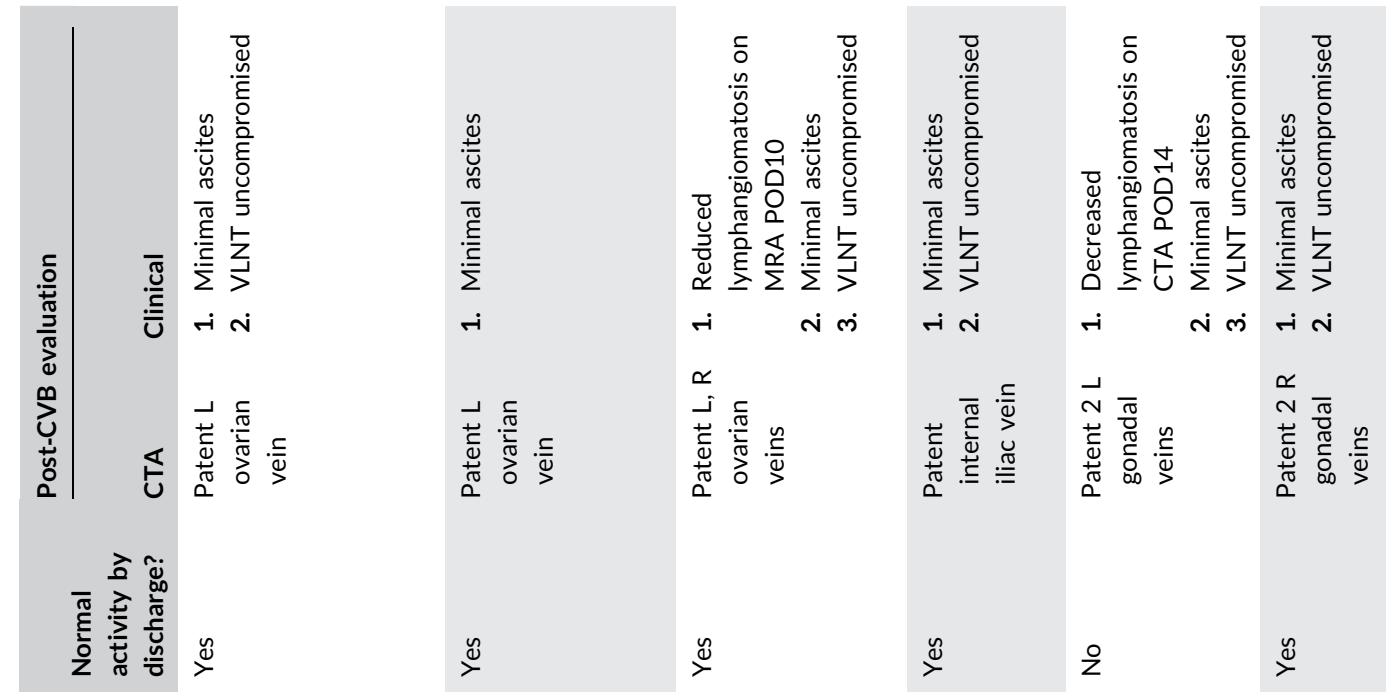

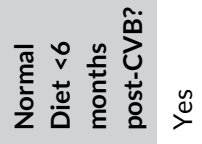
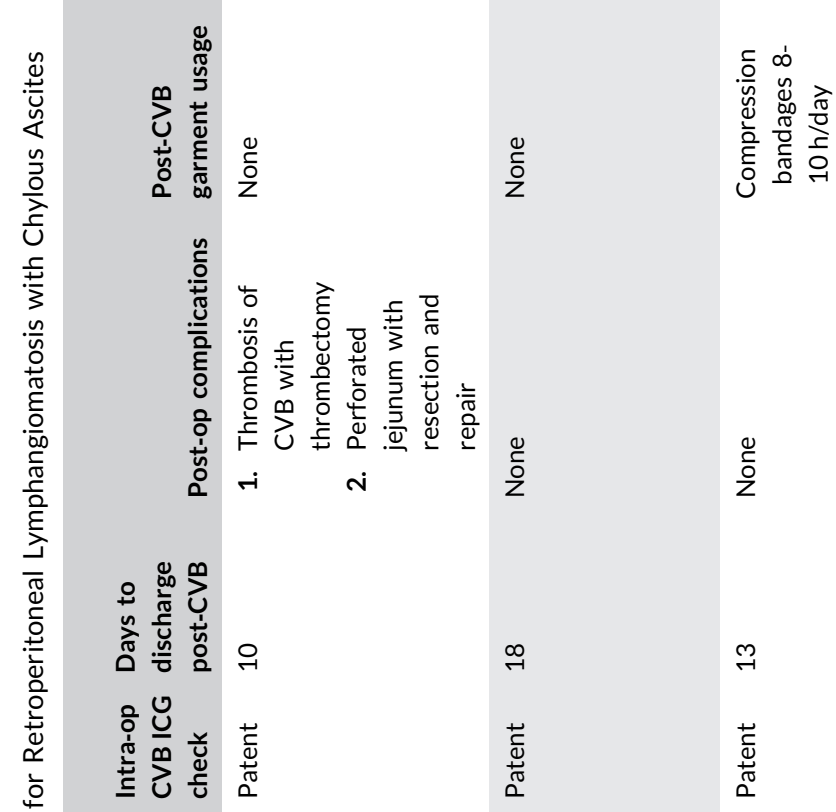

$\stackrel{2}{\check{0}}$
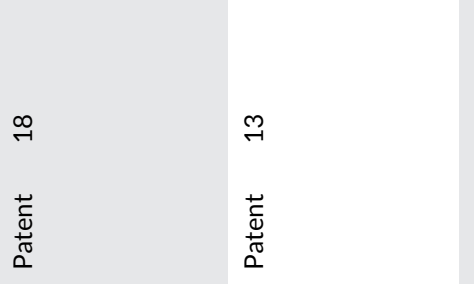

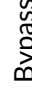

告

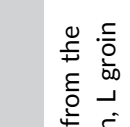

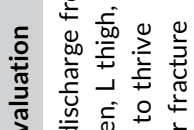

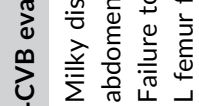

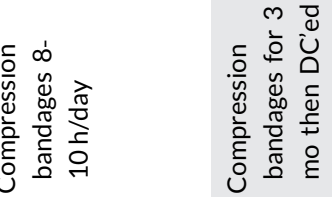

$\succ$
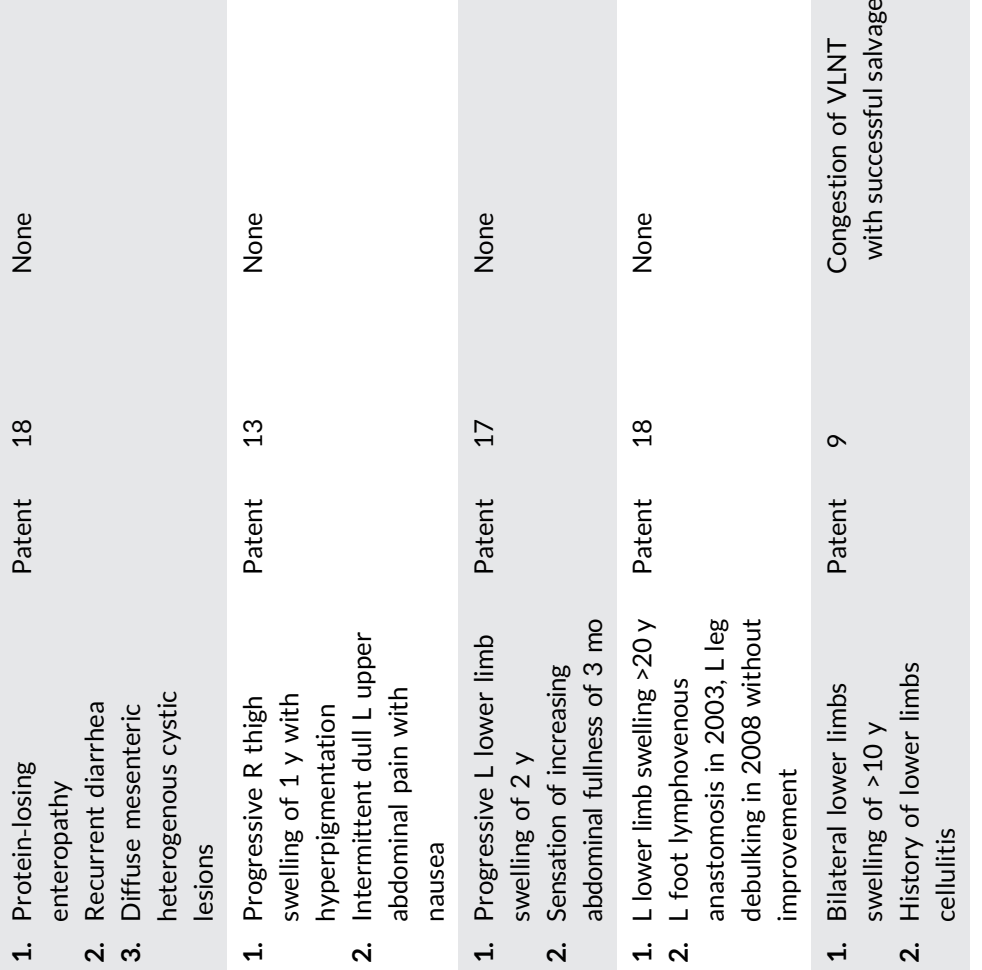


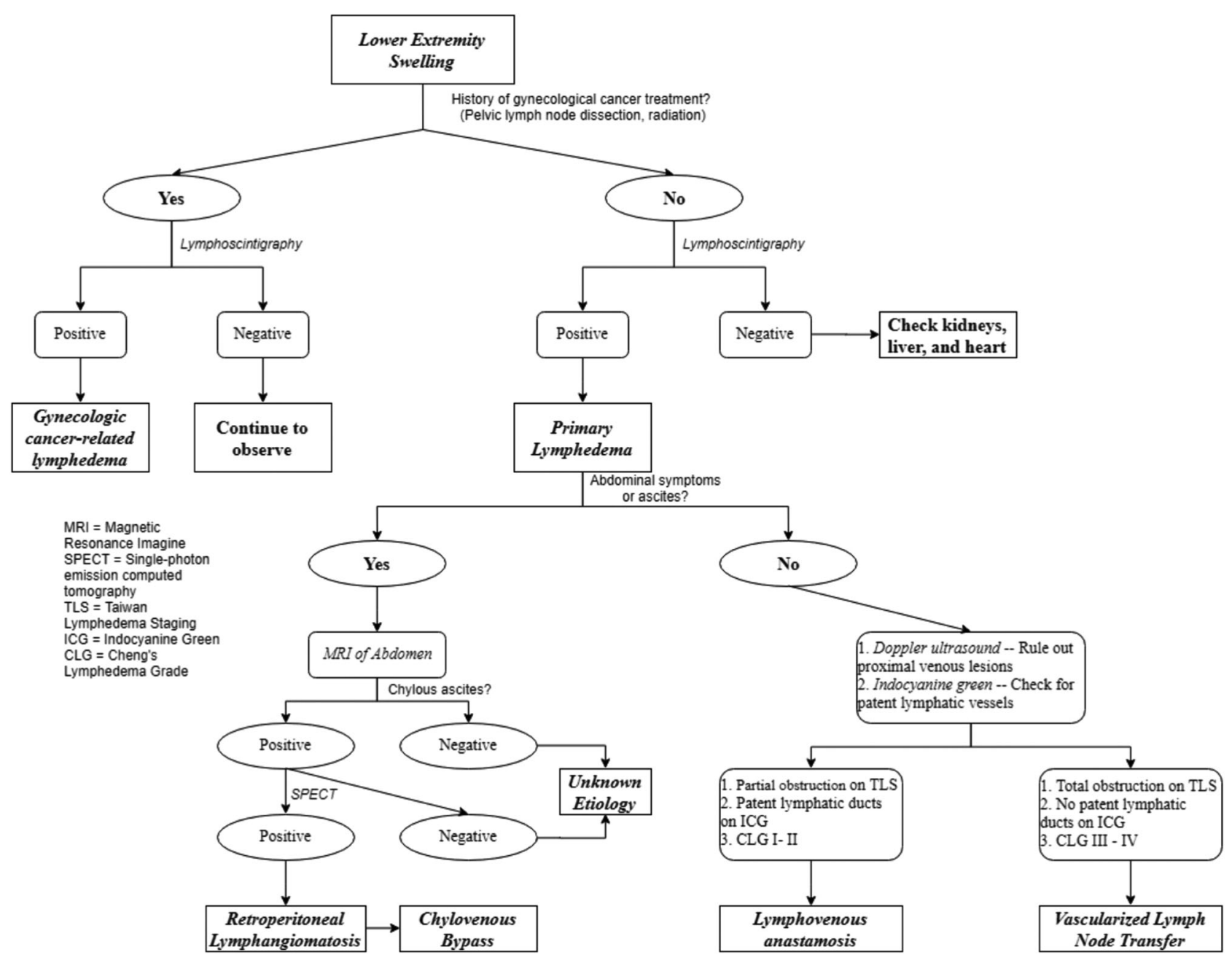

FIGURE 3 Proposed algorithm for the diagnosis and treatment of retroperitoneal lymphangiomatosis stepwise diagnostic pathway for work-up, differential diagnoses of possible causes of lower extremity lymphedema

The single patient in our study unable to discontinue wearing compression garments postoperatively opted to do so for comfort, as her occupation requires her to stand for prolonged periods of time.

A potential mechanism for our observations is that the CVB technique creates a controlled outlet for high-volume chyle leakage from the lymphangiomatosis. This opening enables the drainage of chyle/lymph from the retroperitoneal space directly back to the venous system without passing into the thoracic ducts. Joining the lymphatic system with the systemic venous system thereby functions as a near-physiologic solution that not only prevents ascites but also limits protein loss and excessive lymphatic drainage to the extremities, which could otherwise contribute to hypoalbuminemia, lymphedema, infection, and soft tissue or bone damage.

Interestingly, although CVB theoretically enables the return of lost products to the systemic circulation, not all patients presented with hypoalbuminemia pre-CVB, and not all patient presented an improvement in albumin level post-CVB $(P=0.500)$. The lack of consistent hypoalbuminemia in the series could be a reflection of the duration of chylous ascites, with perhaps normal albumin levels reflecting earlier stages of RL. Persistently low albumin levels at follow-up may be due to slow replenishment after a prolonged loss in the form of chylous ascites.

Patient body weights may decrease after VLNT but increase after CVB. It is difficult to determine the cause of a significant difference when the patients have undergone two procedures. However, the fact that all but one patient could tolerate a normal diet without weight gain should be considered. The $4.6 \mathrm{~kg}$ gain in patient 1 is likely actually a reflection of clinical improvement, as she was underweight at $33.4 \mathrm{~kg}$ as a fully grown adult as a result of her severe, chronic malnourishment from chylous leakage alongside her numerous other disease states.

Although the time between when VLNT and CVB were performed varied, the rate of resolution of the chylous ascites or lower-extremity lymphedema improvement remained unchanged. These results do not indicate that VLNT was unhelpful; however, patients still had decreased chyle output and decreased lower extremity circumference with VLNT alone. Further studies with multivariate component analysis would be better suited to parse the contributions of VLNT vs CVB.

One concern that was later considered by the authors during the course of the series was that the omental lymph node transfer may not 


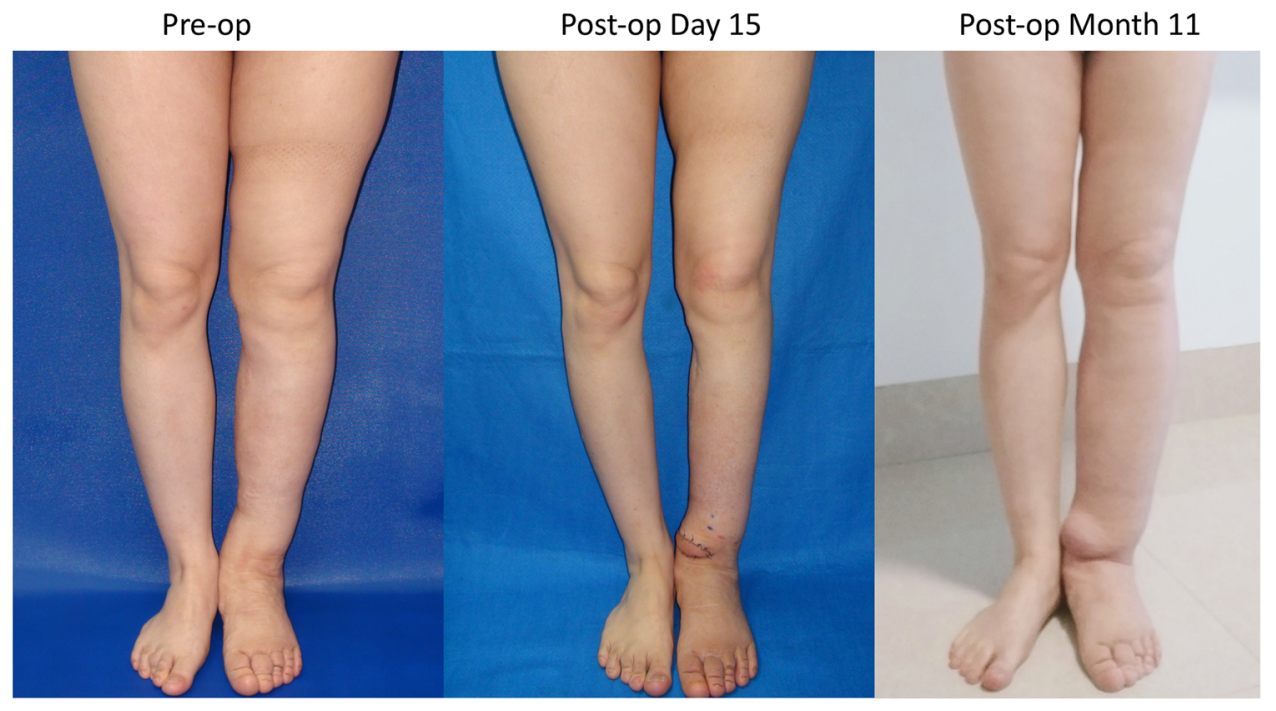

FIGURE 4 Preoperative and postoperative photographs of the retroperitoneal lymphangiomatosis with left lower extremity lymphedema of patient 5. A, Preoperative evaluation of patient 5's left lower extremity immediately after removal of her compression garments, demonstrating characteristic fibro-adipose tissue deposits and lymphedematous enlargement throughout from left thigh to left foot. B, Postoperative day 15 imaging of the patient following her vascularized lymph node transfer and chylovenous bypass, showing reduction in circumference around the region above her knee, below her knee, and over the dorsal aspect of her foot, with protuberance around the ankle largely due to the VLNT. C, Follow-up imaging of the patient's left leg at 11 months with measurements above the knee, below the knee, and above the ankle still slightly improved compared with preoperative measurements despite not wearing compression garments since surgery and standing for long stretches constantly at her occupation. VLNT, vascularized lymph node transfer [Color figure can be viewed at wileyonlinelibrary.com]

necessarily be effective for the extremity lymphedema, as the omental lymph nodes mainly drain the abdominal chyle. If the lower-extremity lymphedema observed was truly due to chylous ascites causing lymphatic leakage into the legs, then the omental VLNT would be ineffective, as drainage of chyle in situ proved inadequate. In patients 4 and 5, the lower extremities underwent lymph node transfer without removing nodes from the abdomen. Patient 2 did not have lowerextremity lymphedema and thus did not undergo VLNT, although it is possible she could have eventually developed lymphedema had her disease not been caught early. The measurements of patient 1 could not be standardized given her left hip disarticulation.

There are many challenges in performing a CVB: coordination with a general surgeon for exploration and reconstructive microsurgeon for anastomosis; a deep, chyle-flooded operative field; intestinal peristalsis causing movement; limited recipient vessels; requirement for longer microsurgical instruments; and having to perform anastomosis of a vein to a literal hole in the wall of the retroperitoneum under a microscope. The anastomosis itself is technically challenging, requiring multiple interrupted sutures while avoiding leakages and controlling internal bleeding. Continuous suturing is inadvisable, as this technique can narrow the anastomosis, resulting in low flow or thrombosis that could lead to inadequate chyle drainage.

Maegawa et al described using vein grafts to resolve cutaneous chyle leakages in the lower limb from the dilated lymphatic duct to the adjacent saphenous vein at two levels. ${ }^{19}$ In comparison, we identified the intraabdominal abnormalities as the source of chylous ascites and chose to perform CVB from the incompetent tissue deep in the abdominal cavity to a low resistance path into the local venous system. By doing so, we bypassed the chyle leak and prevented continued lymphatic output from the lymphangiomas, resulting in complete treatment.

This study also provides a descriptive framework of how one might diagnose RL, particularly if there is no overt primary complaint of chylous ascites (Figure 3). Further studies of the outcomes of CVB on $\mathrm{RL}$ with chylous ascites and lower-extremity lymphedema are needed to validate the operative safety, outcomes, and long-term resolution.

\section{5 | CONCLUSIONS}

RL with chyle leakage and lower-limb lymphedema is a disease that is difficult to diagnose and treat. Lymphoscintigraphy, SPECT, and MRI are the recommended imaging modalities for diagnosing RL in primary lymphedema patients and/or patients with chylous ascites. Although the data are limited, CVB with vascularized lymph node flap transfer is recommended as a first-line measure for effective shunting of chyle into the intraabdominal venous system, simultaneously resolving the secondary lower-extremity lymphedema.

\section{DATA AVAILABILITY}

Raw data were generated at Chang Gung Memorial Hospital. Derived data supporting the findings of this study are available from the corresponding author on request.

\section{CONFLICT OF INTERESTS}

The authors declare that they have no conflict of interests. 


\section{ORCID}

Courtney Chen (D) http://orcid.org/0000-0001-5389-3461

Ming-Huei Cheng (D) http://orcid.org/0000-0002-4237-2959

\section{REFERENCES}

1. Gordon KD, Mortimer PS. Progressive Iymphangiomatosis and Gorham's disease: case report and clinical implications. Lymphat Res Biol. 2011;9:201-204.

2. Blei F. Lymphangiomatosis: clinical overview. Lymph Res Biol. 2011;9:185-190.

3. Aviv RI, McHugh K, Hunt J. Angiomatosis of bone and soft tissue: a spectrum of disease from diffuse lymphangiomatosis to vanishing bone disease in young patients. Clin Radiol. 2001;56:184-190.

4. Gorham LW, Stout AP. Massive osteolysis (acute spontaneous absorption of bone, phantom bone, disappearing bone); its relation to hemangiomatosis. J Bone Joint Surg Am. 1955;37-A:985-1004.

5. Lala S, Mulliken JB, Alomari Al, et al. Gorham-Stout disease and generalized lymphatic anomaly. Skeletal Radiol. 2013;42:917-924.

6. Rockson SG. Diseases of the lymphatic circulation. Vascular Medicine: A companion to Braunwald's Heart Disease. 2013;2e:697-708.

7. Bhardwaj R, Vaziri H, Gautam A, et al. Chylous ascites: a review of pathogenesis, diagnosis, and treatment. J Clin Transl Hepatol. 2018; 6(1):105-113.

8. Campisi C, Bellini C, Eretta C, et al. Diagnosis and management of primary chylous ascites. J Vasc Surg. 2006;43:1244-1248.

9. Kinmonth JB, Taylor GW. Chylous Reflux. Br Med J. 1964;1:529-532.

10. Noel AA, Gloviczki P, Bender CE, Whitley D, Stanson AW, Deschamps C. Treatment of symptomatic primary chylous disorders. J Vasc Surg. 2001;34:785-791.

11. Kinmonth JB. Management of some abnormalities of the chylous return. Proc $R$ Soc Med. 1972;65:721-722.
12. Browse NL, Wilson NM, Russo F, et al. Aetiology and treatment of chylous ascites. Br J Surg. 1992;79:1145-50.

13. Sachanandani NS, Chu SY, Ho OA, et al. Lymphedema and concomitant venous comorbidity in the extremity: comprehensive evaluation, management strategy, and outcomes. J Surg Oncol. 2018;118(6):941-952.

14. Cheng M, Pappalardo M, Lin C, et al. Validity of the Novel Taiwan Lymphoscintigraphy Staging and Correlation of Cheng Lymphedema Grading for Unilateral Extremity Lymphedema. Ann Surgery. 2018;268(3): 513-525.

15. Rockson SG. Lymphedema after breast cancer treatment. N Engl J Med. 2018;379:1937-1944. https://doi.org/10.1056/NEJMcp1803290

16. Cheng $\mathrm{MH}$, Huang JJ, Nguyen $\mathrm{DH}$, et al. A novel approach to the treatment of lower extremity lymphedema by transferring a vascularized submental lymph node flap to the ankle. Gynecol Oncol. 2012;126:93-98.

17. Ito R, Zelken J, Yang CY, et al. Proposed pathway and mechanism of vascularized lymph node flaps. Gynecol Oncol. 2016;141:182-188.

18. Cheng $\mathrm{MH}$, Huang JJ, Wu CW, et al. The mechanism of vascularized lymph node transfer for lymphedema: natural lymphaticovenous drainage. Plast Reconstr Surg. 2014;133:192e-198ee.

19. Maegawa J, Mikami T, Yamamoto $Y$, et al. Lymphaticovenous shunt for the treatment of chylous reflux by subcutaneous vein grafts with valves between megalymphatics and the great saphenous vein. Microsurgery. 2010;30:553-556.

How to cite this article: Chen C, Chu S-Y, Lin C, Liu K-H, Cheng $\mathrm{M}-\mathrm{H}$. Intra-abdominal chylovenous bypass treats retroperitoneal lymphangiomatosis. J Surg Oncol. 2020;121:75-84. https://doi.org/10.1002/jso.25514 